\title{
A method to optimise train energy consumption combining manual energy efficient driving and scheduling
}

\author{
C. Sicre ${ }^{1}$, P. Cucala ${ }^{1}$, A. Fernández ${ }^{1}$, J. A. Jiménez ${ }^{2}$, \\ I. Ribera ${ }^{2} \&$ A. Serrano ${ }^{2}$ \\ ${ }^{I}$ Instituto de Investigación Tecnológica, \\ Escuela Técnica Superior de Ingeniería, \\ Universidad Pontificia Comillas, Spain \\ ${ }^{2}$ Renfe Operadora, Spain
}

\begin{abstract}
This article presents a combined simulation and optimisation technique to optimise the energy consumption of a single manual-driving train service with inter-stations along a High Speed Line. For this purpose, the best energy efficient driving strategies along every stretch between two stations will be simulated with an accurate and detailed train simulator to obtain the run time and energy consumption Pareto curves of each stretch. Afterwards, an optimisation tool will distribute the available slack time for the service among the stretches, which will minimise the overall energy consumption of the service. The potential of the method will be shown with a case study at the end of the article.
\end{abstract}

Keywords: manual energy efficient driving, ecodriving, coasting, timetable, Pareto curves, slack time.

\section{Notation and terminology}

Flat-out time: minimum run time that a train can achieve satisfying all the constraints of a service

Slack time: Time that added to the flat-out time constitutes the commercial run time of a stretch, and whose aim is to ensure punctuality in case of delays

Grade: Longitudinal track inclination, positive uphills, negative downhills, [\%o]

HSL: High Speed Line 
Neutral section: Piece of rail track without electric feeding

Pareto curve: Curve consisting of the drivings that, for a fixed run time, consume the least amount of energy

\section{Introduction}

Reduction of energy consumption on railway systems has recently turned to be a global concern as a contribution to reduce the global warming. Many fields can contribute to this aim, for instance, infrastructure, signalling, maintenance and operation. This paper will focus it from the operation point of view, in particular, by means of manual energy efficient driving and efficient scheduling.

\subsection{Manual efficient driving}

There have been many contributions about manual energy efficient driving. Basically, there are three different ways a train can be driven: applying traction, coasting (no traction effort) or braking. The problem about manual efficient driving is when to switch to each way, fulfilling the constraints of the service.

For modelling the manual driving, different authors have translated the experience of the drivers into different manual driving parameters; in [1], four parameters are defined to build a driver model for calculating energy consumption. In [2], the authors introduce the maximum and minimum coasting speed parameters. With relation to the previous parameters, in [3] it is introduced a distance, measured from a departure station, before it the train is not allowed to coast.

Researches about finding the best strategy to drive a train started on the late 60 s, when the optimal control for a linear train model was solved analytically applying Pontryagin's Maximum principle [4], concluding that the optimal driving strategy consisted of four sections: starting with maximum acceleration, holding speed, coasting, and finally braking with maximum deceleration. This conclusion was reinforced in [5] and [6], with running tests that lowered the energy consumption by $13 \%$ and $20-25 \%$ respectively.

A different approach for finding the best driving is by training the drivers; in "A toda Vela" project [7], a group of AVE (Spanish High Speed) drivers took part in a contest consisting of consuming the least amount of energy along the service Madrid-Sevilla without affecting the punctuality commitment. The results show achievable savings up to $9,5 \%$.

Other attempts to find the best efficient strategy have been focused on calculating the best coasting points along the rail track, like $[3,8,9]$, or determining an optimal holding speed lower than the speed limit, like [10] and [11]. Other techniques deal with the reduction of the unnecessary braking; one of them is explained in [12] and consists of avoiding the use of the brake during sections where the grade would otherwise increase the speed above the desired holding speed. To achieve that purpose, the train must start coasting early enough to reduce the same speed that will be recovered along the downgrade. Another similar technique consists in holding speed without braking, [13]; when 
the train is allowed to increase the holding speed up to the speed limit of the line, it maintains the holding speed always that tractive effort is needed, otherwise, instead of braking, it starts coasting, increasing its speed. The train then can either reach the speed limit, when it will brake in order not to exceed it, or start decreasing its speed due to upgrades, reaching again the holding speed, when tractive effort will be again applied.

\subsection{Design of efficient timetables}

The timetable of the service must be feasibly designed attending to the train, line characteristics and users demand. It is built adding a slack time to the flat-out time, to ensure the punctuality in case of any delays.

There are researches that have been focused on designing slack times taking into account behavioural responses [14]. Others have been focused on minimising interchanges waiting times, like [15], where the authors consider a fixed value of slack time for the whole service and the available headway for each route, distributing it the best way for avoiding delays, or [16], where evolutionary techniques are used for the same purpose.

Related with energy consumption, in [17] the timetable is designed by adding a slack time to the flat-out time with the aim of reducing the energy consumption for a fixed service time. More ambitiously, the work in [18] looks for a compromise between timetable synchronization and energy minimisation.

\section{Model ecodriving - timetable}

The objective of this work is to minimise the global energy consumption of a single train service with inter-stations along a HSL by manual energy efficient driving and timetable design, which is carried out both by a simulation and optimisation models.

A general train service consists of a head station, $n$ inter-stations and a terminal station, resulting in $n+1$ stretches. The purpose of the optimisation model is to distribute the fixed available slack time present in every timetable among the different stretches of a service in the most efficient way. Besides, the aim of the simulation model is finding the most energy efficient drivings along each stretch, calculating run times and energy consumptions with a high grade of accuracy.

A train service is always associated with a timetable, where the commercial time of a stretch is built up by the addition of the flat-out time and the slack time. This slack time is usually calculated as a percentage of the flat-out time plus an additional time that depends on the length of the service, according to UIC Code 451-1. The difference between the slack time and the time delays during a service will be the available time to perform an energy efficient driving.

The optimisation model will distribute the slack time of the service among its $n+1$ stretches minimising the global energy consumption. To achieve it, there are needed the run times and energy consumptions of the best energy efficient drivings of every stretch, and this is extracted from the simulation model. 
The simulator will calculate run times and energy consumptions of manual drivings by the efficient combination of the driving techniques described in the introduction. Each driving is associated with a data pair of run time and energy consumption, so the drivings that, for a given time, consume the least amount of energy, will constitute the so called Pareto curve for that stretch, [19]. This curve relates each run time with the minimum achievable energy consumption, Figure 1.

The results of the simulator are therefore $n+1$ Pareto curves. Those Pareto curves, together with the slack time for the whole service, will be the input data of the optimisation model for distributing the slack time in the best energy efficient way. After obtaining the optimal slack time for each stretch, it will be only necessary to add it to its corresponding flat-out time to obtain the associated commercial time. The most efficient drivings fulfilling the whole service energy optimisation will be located on the Pareto curves. Figure 2 shows a block diagram for the whole process.

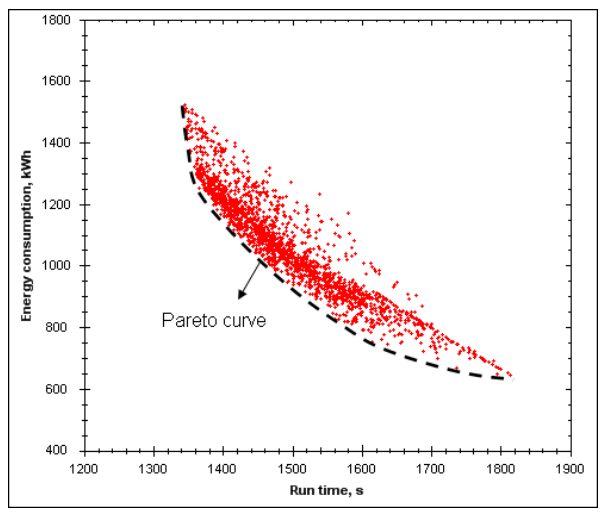

Figure 1: Run time-energy consumption and associated Pareto curve.

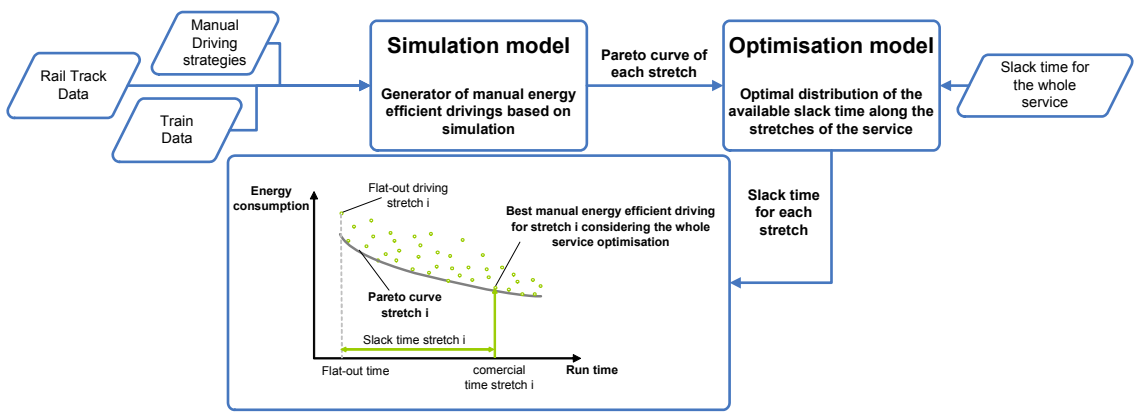

Figure 2: Block diagram for optimising train energy consumption. 


\section{Simulation model}

\subsection{Description of the simulator}

In order to calculate accurately run times and energy consumptions, a detailed step-based simulation model has been developed [20, 21].

The step-based simulation divides the train movement into a succession of intervals which duration/length is the step itself. Each interval is characterised by an initial and a final state, providing that the final state of one step will be the initial state of the following. Step-based simulation is utilised when the train is varying its speed, and its grade of accuracy is inversely proportional to the duration/length of the step, which may be as small as needed, with the constraint of the computational demand. Nevertheless, due to the fact that one of the main requirements of the simulator is calculating run times and energy consumptions accurately, the step must be kept small. During each step, the acceleration is considered as constant (which is a reasonable assumption when the step is small), so the state variables at the end of a step can be calculated from the state variables at the beginning of it and the equations of the uniformly accelerated linear motion:

\section{Time step-based simulation:}

$$
\begin{aligned}
& s_{f}=s_{0}+v_{0} \cdot \Delta t+0.5 \cdot a \cdot \Delta t^{2} \\
& v_{f}=v_{0}+a \cdot \Delta t \\
& t_{f}=t_{0}+\Delta t
\end{aligned}
$$

Being $s_{0}, v_{0}, t_{0}, s_{f}, v_{f}, t_{f}$ the distance, speed and time at the beginning and the end of the step respectively, $a$ the acceleration, and $\Delta t$ the time step.

\section{Space step-based simulation:}

$$
\begin{aligned}
& s_{f}=s_{0}+\Delta s \\
& v_{f}=\sqrt{v_{0}^{2}+2 \cdot a \cdot\left(s_{f}-s_{0}\right)} \\
& t_{f}=t_{0}+\frac{v_{f}-v_{0}}{a}
\end{aligned}
$$

Being $s_{0}, v_{0}, t_{0}, s_{f}, v_{f}, t_{f}$ and $a$ the same as previously, and $\Delta s$ the distance step.

This simulation model is based on object-oriented programming and has been implemented in $\mathrm{C}++$.

\subsection{Input data}

The input introduced into the simulator consists of the three upper left blocks shown in Figure 2: train, infrastructure/service and manual driving strategy.

The train is modelled as a length distributed and includes rotary inertia to account for the effect of rotary masses on acceleration. The maximum electric tractive and braking effort curves are modelled with respect to the speed 


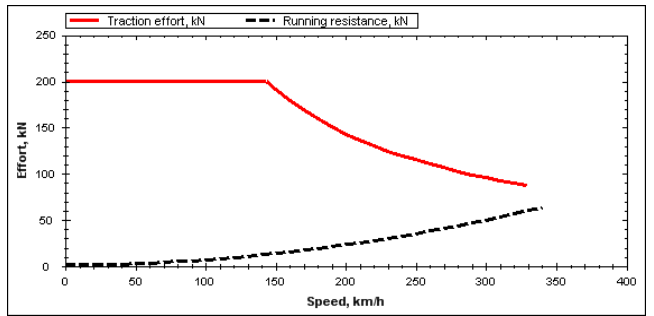

(A)

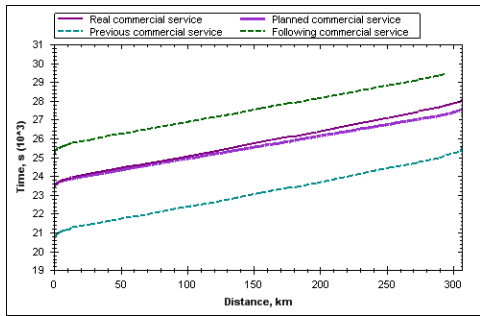

(B)

Figure 3: (A) Traction, braking effort and running resistance curves as a function of speed. (B) Available headway for the commercial service.

(Figure 3A), and their efficiencies may depend both on the speed and the powering/braking ratio, or either be constant. The running resistance is modelled as a second degree polynomial with respect to the speed (Figure 3A). The model also takes into account the consumption of auxiliary equipment and comfort systems and slippage between rail and wheels. It has also the possibility of using regenerative braking and/or onboard energy storage devices.

The infrastructure and the service are defined with the following data:

a. Check points, stopping stations, stopping times and timetable.

b. Speed limits, both permanent and temporary.

c. Grades and grade transitions.

d. Neutral sections, tunnels and track width.

e. Curves, distinguishing among straight sections, curves and clothoids.

f. Headway: it is a space-time margin for a specific service out of which it is not desirable the train to be, to avoid introducing delays in the line (Figure 3B).

To enable the simulation of manual driving strategies, a driver module has been implemented, where different strategies can be managed with a set of configurable parameters and manual efficient driving techniques that were described in the introduction.

The most efficient techniques to reduce the energy consumption are holding speed without braking and performing a coasting process before braking to reach a speed limit or a stopping station. Hence, a typical efficient driving may consist of several sections of different holding speeds combined with a final coasting process, as it is shown in (Figure 4) for the stretch Madrid-Zaragoza, where three different sections of holding speed were combined with a final coasting section before the braking process.

The simulation process for obtaining the best manual driving strategies along a service begins with the definition of the track, train and service data described in the previous sections. The track is divided into the $n+1$ stretches that forms the service studied. Next, a guided search is developed along each stretch independently. This is carried out by combining the manual driving parameters and strategies outlined in the introduction. Each combination configures a driving that will produce a concrete pair of run time and energy consumption. 


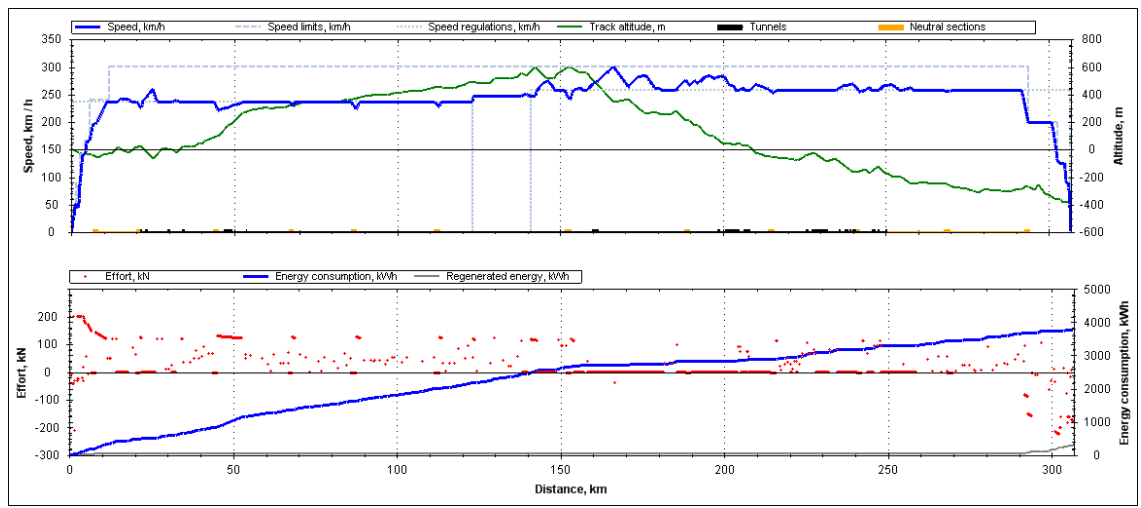

Figure 4: Typical driving consisting of different holding speeds without braking sections and a final coasting section.

One of the main contributions of this simulation search technique is that a wide range of manual driving strategies can be analysed combining different sections of holding speed with different coasting intervals. This richness of definition of manual drivings contrast with the simplification usually found in the literature, which is admissible for short metropolitan services, but not for HSL. Furthermore, calculating energy consumption by an accurate simulator is a guarantee of reliable results. Other methods even avoid calculating energy consumption during the optimisation process, using instead Artificial Neural Networks. Another advantage of this method is that there can be simulated as many strategies as desired, so it will be always possible to find an efficient driving strategy that lasts a desired time.

Once simulated the best manual driving strategies for each stretch, they are obtained $n+1$ distributions like the one shown in Figure 1. The drivings that, for a given run time, provide the minimum energy consumption, will set up the Pareto curve, consisting of the best manual drivings for each run time. The output of the simulation process is the $n+1$ Pareto curves that will be the input to the optimisation model. Whether the train uses regenerative braking, energy storage devices or none of them, the shape of the Pareto curve will be different, so depending on the use of these devices, the optimal driving strategy may vary.

\section{Optimisation model}

The timetable optimisation model will use the Pareto curves and the available slack time for the service to distribute it in the best energy efficient way. It will be assumed that the whole slack time is available for efficient purposes. Each Pareto curve will be modelled as a polygonal whose segments can be adjusted in order to achieve more precision, Figure 5. 


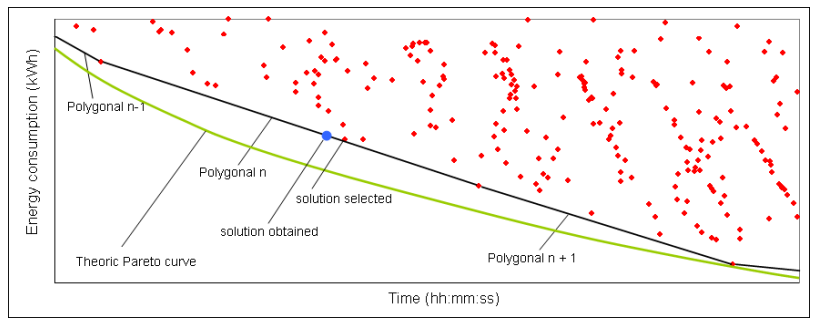

Figure 5: Theoretic and modelled Pareto curves.

The mathematic formulation of the optimisation model is the following:

\section{Indexes}

$i \quad$ Number of stretches along the service, $i=1, \ldots, n+1$

$j \quad$ Number of segments of the polygonal Pareto curve

$n \quad$ Number of inter-stations

\section{Parameters}

$a_{i, j} \quad$ Slope of segment $j$ from the polygonal of stretch $i$

$b_{i, j} \quad$ Ordinate of segment $j$ of the polygonal of stretch $i$

C Slack time for the whole service [s]

$C f_{i} \quad$ Minimum slack time for stretch $i$

$P \quad$ Stopping time at station [s]

$R m_{i} \quad$ Flat-out time for stretch [s]

$R m \quad$ Minimum run time for the whole service [s]

$S_{i, j} \quad$ Equation of segment $j$ of the polygonal of stretch $i$

$R \quad$ Commercial time for the whole service [s]

\section{Variables}

$C_{i} \quad$ Slack time for stretch $i[\mathrm{~s}]$

$R_{i} \quad$ Commercial time for stretch $i[\mathrm{~s}]$

$y_{i, j} \quad$ Indicates activation of segment $j$ from the polygonal of stretch $i$

\section{Equations}

The commercial time of a stretch is the sum of the flat-out and the slack time.

$$
R_{i}=R m_{i}+C_{i}
$$

Equation of each segment $j$ from the polygonal of stretch $i$.

$$
S_{i, j}=a_{i, j} \cdot R_{i}+b_{i, j}
$$

The commercial time of the service is equal to the addition of the commercial times of each stretch and the stopping time at the inter-stations.

$$
R=\sum_{i=1}^{n+1} R_{i}+n \cdot P
$$


The sum of slack times is equal to the slack time for the whole service.

$$
C=\sum_{i=1}^{n+1} C_{i}
$$

Slack times must be bigger than a certain fixed value to guarantee punctuality.

$$
C_{i} \geq C f_{i}
$$

Energy consumption of stretch $i$.

$$
E_{i}=\sum_{j=1} y_{i, j} \cdot\left(a_{i, j} \cdot\left(R m_{i}+C_{i}\right)+b_{i}\right)
$$

Only one segment in each Pareto curve is activated.

$$
\sum_{j=1} y_{i, j}=1
$$

The objective function consists in the minimisation of the total amount of energy

$$
\min \sum_{i=1}^{n+1} \sum_{j=1}^{j} y_{i, j} \cdot\left(a_{i, j} \cdot\left(R m_{i}+C_{i}\right)+b_{i}\right)
$$

The way the Pareto curves are defined ensures that the solution obtained is achievable; $n$ out of $n+1$ commercial times will be located at the junction of two segments of the modelled Pareto curves. The position of the last one will be inside a segment, which may not coincide with a simulated driving. In that case, it will be selected the most similar one, (Figure 5).

The optimisation model has been implemented in Gams, and the result is the slack time to be added to each of the $n+1$ flat-out times, what will determine the commercial time of the $n+1$ drivings that minimise the energy consumption of the whole service.

\section{Case study}

To evaluate the effectiveness of the exposed method, a real case will be presented, consisting on a service operated by Renfe, which will be MadridZaragoza, with stops at Guadalajara and Calatayud, and developed by the train Talgo-Bombardier Class-102. Firstly, there are simulated the optimal manual drivings along its three stretches, obtaining the Pareto curves for MadridGuadalajara, Guadalajara-Calatayud and Calatayud-Zaragoza. Finally, there is proposed a redistribution of the available slack time among the three stretches in order to optimise the global energy consumption.

The timetable has a fixed commercial duration $R_{i}$ for each of the three stretches. Simulating the flat-out driving for the Class-102 on each of them they are obtained the flat-out times $R m_{i}$ and the slack times $C_{i}$, so the sum of the three slack times is the available slack time for the whole service $C$. All these values are shown on the left side of 0 , as well as the energy consumption reached with the flat-out driving.

Now there are simulated the efficient manual driving strategies for each stretch in order to obtain their Pareto curves (Figure 6).

The three Pareto curves are introduced in the optimisation model together with the available slack time for the service $(0: 10: 30)$. The new optimal distribution of slack times and the savings got by this method are shown on the 

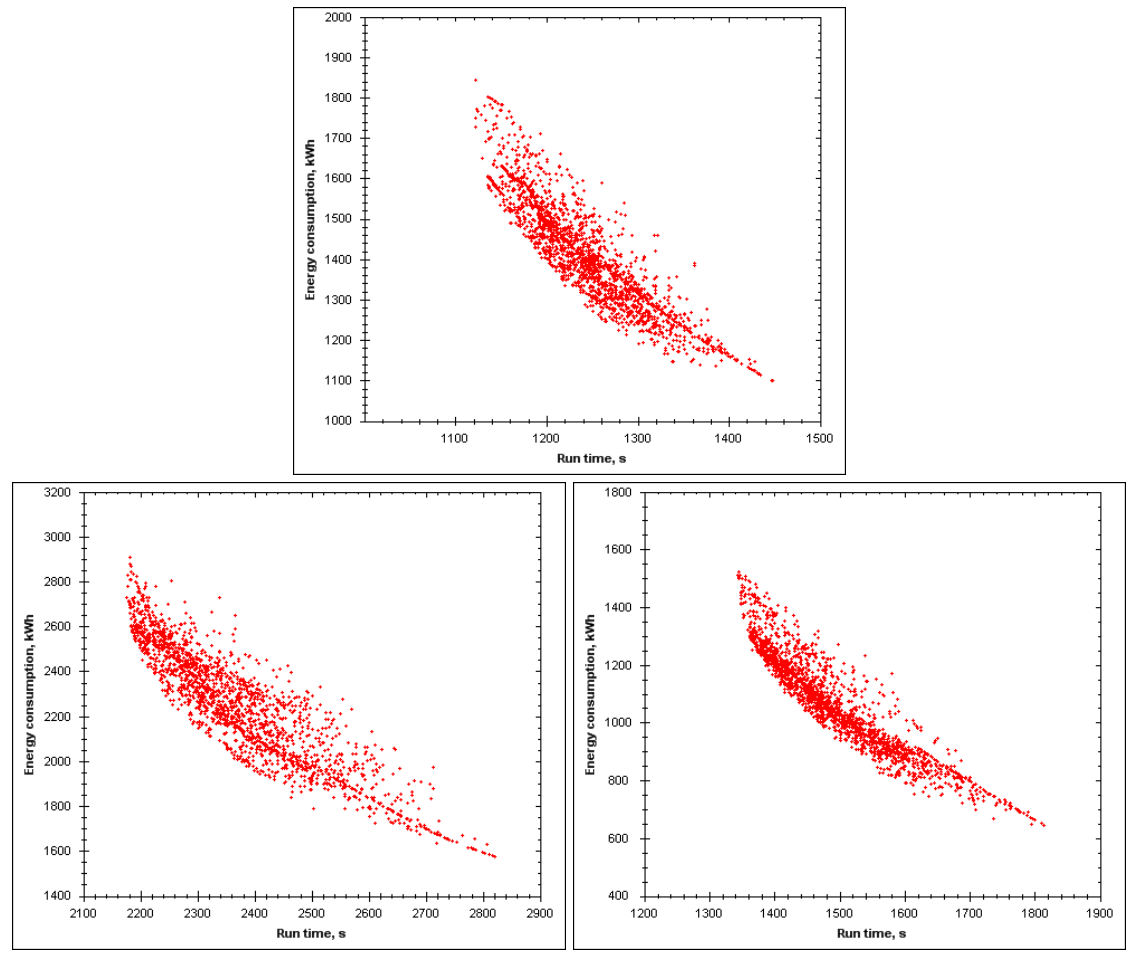

Figure 6: Simulated driving strategies (Top: Madrid-Guadalajara. Left bottom: Guadalajara-Calatayud. Right bottom: CalatayudZaragoza).

Table 1: Results of run times and energy consumptions for commercial timetable and optimised timetable.

\begin{tabular}{|c|c|c|c|c|c|c|c|c|}
\cline { 2 - 9 } \multicolumn{1}{c|}{} & \multicolumn{4}{c|}{ Commercial timetable with flat-out driving } & \multicolumn{3}{c|}{ Optimised timetable with energy efficient driving } \\
\cline { 2 - 9 } & $\begin{array}{c}\text { Commercial } \\
\text { timetable } \\
\text { Ri }\end{array}$ & $\begin{array}{c}\text { Flat-out time } \\
\text { Rmi } \\
\text { hh:mm:ss }\end{array}$ & $\begin{array}{c}\text { Slack } \\
\text { time } \\
\text { hh:mm:ss }\end{array}$ & $\begin{array}{c}\text { Energy } \\
\text { consumption } \\
\text { flat-out } \\
\text { kWh }\end{array}$ & $\begin{array}{c}\text { Designed } \\
\text { timetable } \\
\text { Ri } \\
\text { hh:mm:ss }\end{array}$ & $\begin{array}{c}\text { Designed } \\
\text { slack } \\
\text { time } \\
\text { hh:mm:ss }\end{array}$ & $\begin{array}{c}\text { Optimised } \\
\text { energy } \\
\text { consumption } \\
\text { kWh }\end{array}$ & $\begin{array}{c}\text { Energy } \\
\text { savings } \\
\%\end{array}$ \\
\hline Madrid-Guadalajara & $00: 23: 00$ & $00: 18: 48$ & $00: 04: 12$ & 1690,826 & $00: 21: 04$ & $00: 02: 15$ & 1245,029 & $26,37 \%$ \\
Guadalajara-Calatayud & $00: 39: 00$ & $00: 36: 15$ & $00: 02: 45$ & 2730,136 & $00: 41: 04$ & $00: 04: 49$ & 1835,773 & $32,76 \%$ \\
Calatayud-Zaragoza & $00: 26: 00$ & $00: 22: 26$ & $00: 03: 34$ & 1445,056 & $00: 25: 52$ & $00: 03: 26$ & 841,643 & $41,76 \%$ \\
\hline Total & $01: 28: 00$ & $01: 17: 30$ & $00: 10: 30$ & 5866,018 & $01: 28: 00$ & $00: 10: 30$ & 3922,445 & $33,63 \%$ \\
\hline
\end{tabular}

right side of 0 , which fixes the new optimised timetable that minimises the energy consumption of the whole service. It must be noted that the saving predictions are compared to the flat-out drivings, which are the least efficient ones, but it has been checked on real tests that this is the most typical driving developed by the drivers, whose only current concern is the punctuality of the service. It must also be noted that those savings are obtained only with minimal changes on the timetable (the time for the overall service maintained) as only the 
arrival and departure time of the inter-stations are modified, and performing an efficient manual driving, which are actions that cost no money at all.

\section{Conclusions}

This work has proposed a method based on simulation and optimisation to minimise the energy consumption of a single train service with intermediate stops along a HSL following manual energy efficient driving and efficient scheduling.

The fully detailed simulator guarantees accurate and trustful results of run time and energy consumption of the drivings simulated, and the way the manual driving strategies are defined allows to explore almost all the feasible driving strategies that can be developed by human drivers, allowing a complete search of the best manual energy efficient driving for an isolated stretch.

With the optimal distribution of the available slack time along the different stretches of a service, it is possible to guarantee not only the best efficiency for each of its stretches separately, but the least energy consumption for the whole service.

\section{References}

[1] Lukaszewicz, P., et al. Driving techniques and strategies for freight trains. In Computers in Railways VII. Seventh International Conference on Computers in Railways. COMPRAIL 2000. 2000: WIT Press.

[2] Bocharnikov, Y.V., et al., Optimal driving strategy for traction energy saving on DC suburban railways. IET Electric Power Applications, 2007. 1(5): p. 675 .

[3] Acikbaas, S. and M.T. Soylemez, Coasting point optimisation for mass rail transit lines using artificial neural networks and genetic algorithms. IET Electric Power Applications, 2008. 2(3): p. 172.

[4] Ichikawa, K., Application of optimization theory for bounded state variable problems to the operation of train. Bulletin of the Japan Society of Mechanical Engineers, 1968. 11(47): p. 857.

[5] Yasukawa, S., et al., Development of an on-board energy saving train operation system for the Shinkansen Electric Railcars. Quarterly Report of the Railway Technical Research Institute, 1987. 28(2-4): p. 54.

[6] Van Dongen, L.A.M. and J.H. Schuit. Energy-efficient driving patterns in electric railway traction. In International Conference on Main Line Railway Electrification (Conf. Publ. no.312). 1989: IEE.

[7] Renfe, A.v., A toda vela, la conducción económica. Aprovechamiento máximo de la energía de tracción. 2004.

[8] Chang, C.S. and S.S. Sim, Optimising train movements through coast control using genetic algorithms. IEE Proceedings - Electric Power Applications, 1997. 144(1): p. 65.

[9] Howlett, P., Optimal strategies for the control of a train. Automatica, 1995(4): p. 519-532. 
[10] Pudney, P. and P. Howlett, Optimal driving strategies for a train journey with speed limits. J Austral Math Soc Ser B, 1994. 36: p. 38-49.

[11] Liu, R. and I.M. Golovitcher, Energy-efficient operation of rail vehicles. Transportation Research Part A: Policy \& Practice, 2003. 37(10): p. 917.

[12] Lagos, M., et al. Computer-aided train operation: CATO. In Computers in Railways VII. Seventh International Conference on Computers in Railways. COMPRAIL 2000. 2000: WIT Press.

[13] Hee-Soo, H., Control strategy for optimal compromise between trip time and energy consumption in a high-speed railway. IEEE Transactions on Systems, Man \& Cybernetics, Part A (Systems \& Humans), 1998. 28(6): p. 791.

[14] Carey, M., Optimizing scheduled times, allowing for behavioural response. Transportation Research: Part B, 1998. 32B(5): p. 329.

[15] Golshani, F. and T. Thomas, Optimal distribution of slack-time in schedule design. Traffic Engineering \& Control, 1981. 22(8-9): p. 490.

[16] Chung Min, K. and C.S. Chang, Timetable Synchronization of Mass Rapid Transit System Using Multiobjective Evolutionary Approach. IEEE Transactions on Systems, Man \& Cybernetics: Part C - Applications \& Reviews, 2008. 38(5): p. 636.

[17] Lancien, D. and M. Fontaine, Computing train schedules to save energy. Revue General des Chemins de Fer, 1981. 100: p. 679.

[18] Albrecht, T., et al. A new integrated approach to dynamic schedule synchronization and energy-saving train control. In Computers in Railways. Eighth International Conference. 2002: WIT Press.

[19] Domínguez, M., et al., Computer-aided design of ATO speed commands according to energy consumption criteria. Computers in Railways XI Computer System Design and Operation in the Railway and Other Transit Systems, 2008. 103: p. 183-192.

[20] Law, A.M. and W.D. Kelton, Simulation Modeling and Analysis. Third edition ed. 2000, New York: McGraw-Hill.

[21] Goodman, C.J., L.K. Siu, and T.K. Ho. A review of simulation models for railway systems. In International Conference on Developments in Mass Transit Systems. 1998: IEE. 